\title{
Evaluating Racial/Ethnic Differences in Care Escalation Among COVID-19 Patients in a Home-Based Hospital
}

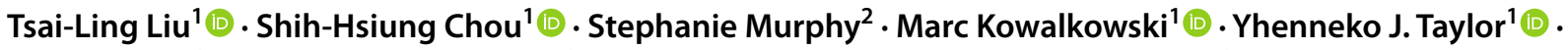

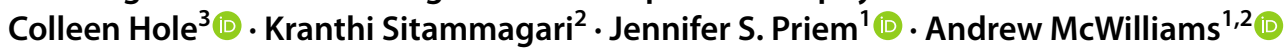

Received: 24 September 2021 / Revised: 16 February 2022 / Accepted: 19 February 2022 / Published online: 7 March 2022

(c) W. Montague Cobb-NMA Health Institute 2022

\begin{abstract}
The novel coronavirus disease 2019 (COVID-19) has infected over 414 million people worldwide with 5.8 million deaths, as of February 2022. Telemedicine-based interventions to expand healthcare systems' capacity and reduce infection risk have rapidly increased during the pandemic, despite concerns regarding equitable access. Atrium Health Hospital at Home $(\mathrm{AH}-\mathrm{HaH})$ is a home-based program that provides advanced, hospital-level medical care and monitoring for patients who would otherwise be hospitalized in a traditional setting. Our retrospective cohort study of positive COVID-19 patients who were admitted to $\mathrm{AH}-\mathrm{HaH}$ aims to investigate whether the rate of care escalation from $\mathrm{AH}-\mathrm{HaH}$ to traditional hospitalization differed based on patients' racial/ethnic backgrounds. Logistic regression was used to examine the association between care escalation within 14 days from index $\mathrm{AH}-\mathrm{HaH}$ admission and race/ethnicity. We found approximately one in five patients receiving care for COVID-19 in AH-HaH required care escalation within 14 days. Odds of care escalation were not significantly different for Hispanic or non-Hispanic Blacks compared to non-Hispanic Whites. However, secondary analyses showed that both Hispanic and non-Hispanic Black patients were younger and with fewer comorbidities than non-Hispanic Whites. The study highlights the need for new care models to vigilantly monitor for disparities, so that timely and tailored adaptations can be implemented for vulnerable populations.
\end{abstract}

Keywords Care escalation $\cdot$ Racial/ethnic $\cdot$ COVID-19 $\cdot$ Home-based hospital

\section{Introduction}

Over 414 million people have been infected with the novel coronavirus disease (COVID-19) globally [1]. Approximately one in five of these reported infections and one in six reported deaths (i.e., over 920,000) have occurred in the USA, as of February 2022 [2]. Throughout the COVID-19 pandemic, racial and ethnic disparities have been widely observed in COVID-19 testing, hospitalizations, and health outcomes [3-9]. Specifically, literature shows non-Hispanic Blacks and Hispanics had a higher incidence of COVID-19,

Tsai-Ling Liu

Tsai-Ling.Liu@atriumhealth.org

1 Center for Outcomes Research and Evaluation (CORE), Atrium Health, 1300 Scott Ave, Charlotte, NC 28204, USA

2 Division of Hospital Medicine, Department of Internal Medicine, Atrium Health, Charlotte, NC, USA

3 Population Health, Atrium Health, Charlotte, NC, USA and higher rates of hospitalization and in-hospital mortality compared to non-Hispanic Whites [10-13]. In addition, racial/ethnic disparities in access to telemedicine services have been reported among patients with chronic diseases, which may have far-reaching consequences given the prominent role telemedicine has played during this pandemic [14]. Outside of the hospital setting, telemedicinebased interventions like hospital at home have been leveraged to rapidly increase health system capacity and access to services, while reducing infection risk. However, experts have diverging opinions regarding the effects of such care models on health disparities $[15,16]$. Thus, the question of whether telemedicine effectively reached populations at risk for health disparities, especially in acute care, remains unanswered.

Atrium Health is one of the 85 healthcare systems approved for providing Acute Hospital Care at Home through the Centers for Medicare \& Medicaid Services Acute Hospital Care at Home Program [17]. The program aims to help hospitals expand access in the face of capacity 
issues related to COVID-19. Atrium Health Hospital at Home (AH-HaH) provides advanced, hospital-level medical care and monitoring for patients, who would otherwise be hospitalized in a traditional setting, through $24 / 7$ virtual nursing, daily virtual provider visits, and twice daily in-home Community Paramedicine visits [18]. Our prior research showed that majority of COVID-19 patients admitted to AH-HaH did not require care escalation to conventional brick-and-mortar hospitals [19]. This study further investigates whether the rate of care escalation differed based on patients' racial/ethnic backgrounds.

\section{Methods}

\section{Setting and Data Source}

This retrospective study used electronic medical record (EMR) data at Atrium Health, a large integrated healthcare system based in Charlotte, NC. Atrium Health has more than 1500 care locations across North Carolina, South Carolina, and Georgia. The EMR system contains data for more than 10 million patient visits annually.

\section{Study Sample}

The study cohort consisted of 2031 adults ( $\geq 18$ years) who were admitted to $\mathrm{AH}-\mathrm{HaH}$ from ambulatory care (note, direct referrals from ambulatory care to $\mathrm{AH}-\mathrm{HaH}$ were discontinued at the beginning of March 2021), emergency departments, or community settings within 14 days of initial positive COVID-19 test (nasopharyngeal swab via polymerase chain reaction [PCR] testing) between March 23, 2020, and September 30, 2021. The enrollment eligibility criteria included patients who resided in Greater Charlotte area with safe living situations, a working phone, and clinical stability at admission (e.g., vital signs suitable for home monitoring, receiving $\leq 4 \mathrm{l} / \mathrm{min}$ supplemental oxygen, no anticipated imaging or invasive procedures within $48 \mathrm{~h}$, and at baseline mental status) [18]. Only the first AH-HaH encounter was included if a patient had multiple visits during the study period. Patients who died in AH-HaH $(n=0)$ or who died after AH-HaH discharge but within 14 days of $\mathrm{AH}-\mathrm{HaH}$ admission $(n=7)$ were excluded, resulting in a final sample of 2024 patients (Fig. 1).

Fig. 1 Selection of study cohort

2,373 adult ( $\geq 18$ years old) patients admitted to hospital at home $(\mathrm{HaH})$ through ambulatory care, emergency departments, or community settings between March 23, 2020 and September 30, 2021 having testing for COVID-19

Exclude patients whose death date was before index admission date $(\mathrm{n}=1 ; 0.04 \%)$

2,372 patients who are alive or death date was after index admission date
Exclude patients who tested negative for COVID-19 $(n=341 ; 14 \%)$

2,031 patients testing positive for COVID-19

Exclude patients who died in $\mathrm{HaH}(\mathrm{n}=0)$

or died within 14 days from $\mathrm{HaH}$

admission without being hospitalized after

$\mathrm{HaH}$ discharge $(\mathrm{n}=7 ; 0.3 \%)$

2,024 patients are eligible for primary study inclusion 


\section{Measures}

The primary outcome was care escalation, defined as direct transfer from $\mathrm{AH}-\mathrm{HaH}$ to a brick-and-mortar facility (also referred to as "traditional hospitalization") within 14 days of index $\mathrm{AH}-\mathrm{HaH}$ admission-similar to our prior research [19]. If care is escalated, the patient was not well-controlled during the hospitalization and requires additional support to maintain their health status. Patient demographics included age, gender, race/ethnicity, and marital status. Race/ethnicity was captured from predefined fields in the EMR that were self-reported by patients. Patients' body mass index, Charlson Comorbidity Index (CCI), most recent oxygen saturation prior to $\mathrm{AH}-\mathrm{HaH}$ admission, and baseline healthcare utilization (i.e., number of inpatient, outpatient, and emergency department [ED] visits within 1 year prior to the index date) were included as covariates. Total minutes travel-time to the closest hospital and Area Deprivation Index (ADI) [20] were linked to each patient's record based on the addresses in the AH enterprise data warehouse. ADI [21] is a factor-based index that incorporates 17 indicators of poverty, educational attainment, and housing quality at the census tract level using the American Community Survey 5-year 2013-2017 estimates [22]. We grouped ADIs into quartiles (Q1-Q4), with Q1 representing affluent communities with the lowest level of exposure to social and health risk factors.

\section{Statistical Analysis}

Sample characteristics were reported as percentages or medians as appropriate. Chi-square tests and $t$-tests were used for bivariate analyses. Logistic regression was used to examine the association between care escalation within 14 days from index $\mathrm{AH}-\mathrm{HaH}$ admission and race/ethnicity. Models were adjusted for age, gender, marital status, CCI, baseline healthcare utilization, and neighborhood characteristics based on census tract (travel time in minutes to the closest hospital and ADI). Odds ratios (OR) and 95\% confidence intervals (95\% CIs) were reported. In exploratory analyses, we applied descriptive statistics to examine the distributions of patient characteristics within race/ethnicity and age strata. $P$-values $<0.05$ were considered statistically significant. Analyses were conducted using SAS version 9.4 (SAS Institute, Cary, NC).

\section{Results}

Among 2024 eligible patients with COVID-19 who were admitted to $\mathrm{AH}-\mathrm{HaH}$ during the study period, $49.7 \%$ were female, with median age of 56 years and median CCI score of 2. More than one-half (52.3\%) were non-Hispanic White, $30.6 \%$ were non-Hispanic Black, and $11.4 \%$ were Hispanic.
There were 369 (18.2\%) patients who required care escalation within 14 days. Among those requiring care escalation, $54.1 \%$ were non-Hispanic White, $27.5 \%$ non-Hispanic Black, and 13.0\% Hispanic (Table 1). Additionally, 47.0\% were female, $56.2 \%$ married, and the median CCI score was 3. In adjusted analyses, the odds of care escalation from AHHaH were lower among non-Hispanic Blacks (OR 0.84, 95\% CI: $0.61-1.00, p=0.052$ ) but higher among Hispanics (OR $1.34,95 \%$ CI: $0.99-1.81, p=0.055)$ compared to non-Hispanic Whites, though odds of care escalation in both groups were not statistically significant. Additionally, patients who were older, with a higher number of ED visits in the 1 year prior to $\mathrm{AH}-\mathrm{HaH}$ admission, and whose ADI was in the third quartile (relative to the first quartile) were more likely to have care escalated to a brick-and-mortar hospital within 14 days than others. Conversely, patients with a CCI score of 1-2 (relative to 0 ) were less likely to have care escalation (Fig. 2).

In exploratory analyses stratified by race/ethnicity, we found non-Hispanic White patients were older and frequently lived in more affluent communities (ADI quartiles 1 and 2); non-Hispanic Black patients were often obese (31\% with Obesity Class III) and lived in more socioeconomically disadvantaged communities (ADI quartile 4); Hispanic patients were younger, with fewer comorbidities, but living in more socioeconomically disadvantaged areas (ADI quartile 4) (Table 2). Additionally, we observed associations between older age at admission and care escalation within non-Hispanic White and non-Hispanic Black subgroups, but not in Hispanic patients. Non-Hispanic White patients who required care escalation also more frequently had high comorbidity burden ( $\mathrm{CCI}=3-4$ or $\geq 5$ ) and high area level deprivation (ADI quartile 3 or 4) compared to those who did not require care escalation. Similar associations were not observed to be statistically significant within non-Hispanic Black or Hispanic subgroups. When stratified by age at AH$\mathrm{HaH}$ admission, older patients were mostly non-Hispanic White, female, less obese, but with more comorbidities, compared to patients less than 65 years old (Table 3). Older patients who required care escalation more frequently had high comorbidity burden ( $\mathrm{CCI}=3-4$ or $\geq 5$ ), high area level deprivation (ADI quartile 3 or 4), and baseline ED utilization, compared to those who did not require care escalation. Similar associations were not observed to be statistically significant within patients $<65$ years of age at $\mathrm{AH}-\mathrm{HaH}$ admission.

\section{Discussion}

Our study adds to the literature on the the impact of telemedicine on racial/ethnic health disparities by examining differences in care escalation among adults with COVID-19 
Table 1 Characteristics of patients with COVID-19 who received AH-HaH care

\begin{tabular}{|c|c|c|c|c|}
\hline & \multirow[t]{2}{*}{ Overall } & \multicolumn{2}{|c|}{ Care escalation within 14 days } & \multirow[t]{2}{*}{$p$-value } \\
\hline & & No & Yes & \\
\hline$N(\%)$ & 2024 & $1655(81.8)$ & $369(18.2)$ & \\
\hline Age at admission, median [IQR], years & $56[44,66]$ & $55[44,65]$ & $59[49,69]$ & $<0.001$ \\
\hline Female, $n(\%)$ & $1006(49.7)$ & $833(50.3)$ & $173(46.9)$ & 0.250 \\
\hline Race/ethnicity, $n(\%)$ & & & & 0.667 \\
\hline Non-Hispanic White & $1059(52.3)$ & $859(51.9)$ & $200(54.2)$ & \\
\hline Non-Hispanic Black & $620(30.6)$ & $514(31.1)$ & $106(28.7)$ & \\
\hline Hispanic & $230(11.4)$ & $185(11.2)$ & $45(12.2)$ & \\
\hline Other or not report & $115(5.7)$ & $97(5.9)$ & $18(4.9)$ & \\
\hline Married, $n(\%)$ & $1161(57.4)$ & $956(57.8)$ & $205(55.6)$ & 0.450 \\
\hline Body mass index, $n(\%)$ & & & & 0.855 \\
\hline Not obese & $751(37.1)$ & $611(36.9)$ & $140(37.9)$ & \\
\hline Obesity Class I & 585 (28.9) & $485(29.3)$ & $100(27.1)$ & \\
\hline Obesity Class II & $315(15.6)$ & $257(15.5)$ & $58(15.7)$ & \\
\hline Obesity Class III & $373(18.4)$ & $302(18.2)$ & $71(19.2)$ & \\
\hline Charlson Comorbidity Index, $n(\%)$ & & & & $<0.001$ \\
\hline 0 & $866(42.8)$ & $726(43.9)$ & $140(37.9)$ & \\
\hline $1-2$ & $759(37.5)$ & $635(38.4)$ & $124(33.6)$ & \\
\hline $3-4$ & $230(11.4)$ & $170(10.3)$ & $60(16.3)$ & \\
\hline$\geq 5$ & $169(8.3)$ & $124(7.5)$ & $45(12.2)$ & \\
\hline Area Deprivation Index, $n(\%)$ & & & & 0.009 \\
\hline Quartile 1 & $503(24.9)$ & $427(25.8)$ & $76(20.6)$ & \\
\hline Quartile 2 & $534(26.4)$ & $450(27.2)$ & $84(22.8)$ & \\
\hline Quartile 3 & $465(23.0)$ & $363(21.9)$ & $102(27.6)$ & \\
\hline Quartile 4 (highest deprivation) & $522(25.8)$ & $415(25.1)$ & $107(29.0)$ & \\
\hline Total minutes travel to the closest hospital, median [IQR] & $15.5[11.5,21.6]$ & $15.5[11.5,21.7]$ & $15.4[11.5,21.5]$ & 0.634 \\
\hline Most recent $\mathrm{O}_{2}$ saturation, median [IQR] & $96[94,97]$ & $96[94,97]$ & $96[94,97]$ & 0.712 \\
\hline \multicolumn{5}{|l|}{ Baseline healthcare utilization*, median [IQR] } \\
\hline Inpatient visits & $0[0,0]$ & $0[0,0]$ & $0[0,0]$ & 0.004 \\
\hline Outpatient visits & $0[0,2]$ & $0[0,2]$ & $0[0,3]$ & 0.014 \\
\hline Emergency department visits & $0[0,1]$ & $0[0,1]$ & $1[0,1]$ & 0.009 \\
\hline
\end{tabular}

Abbreviation: $I Q R$, interquartile range

*Baseline healthcare utilization includes all visits within 1 year prior to the index date

admitted to AH-HaH. Notably, we did not find significant differences in care escalation between Hispanic or nonHispanic Black patients compared to non-Hispanic White patients. However, we found that $\mathrm{AH}-\mathrm{HaH}$ patients who were older, who had more baseline ED visits at admission, and who were living in more socioeconomically disadvantaged neighborhoods (ADI quartile 3 ) had a higher risk of transfer to a traditional hospital. These findings are consistent with other research showing that both clinical and socioeconomic factors contribute to observed disparities in COVID-19 outcomes [11].

Our findings from this large, racially and ethnically diverse cohort of patients who received hospital-level care at home have important implications for future patient selection and delivery of acute care at home. First, although we did not find statistically significant differences in care escalation from $\mathrm{AH}-\mathrm{HaH}$ to brick-and-mortar facilities for Hispanic vs. non-Hispanic White patients, other studies of patients hospitalized in traditional care settings indicate that Hispanics have been disproportionately impacted by poor COVID-19 outcomes [9, 10, 23]. Similar studies have shown that Hispanic patients have higher rates of hospitalization, severe disease requiring intensive care, intubation, and mortality [24-27].

The reasons why our study did not show a significant difference in care escalation for Hispanic patients despite documented disparities in outcomes in other settings are unclear, but may be explained in part by their younger age and fewer comorbidities - both of which have been identified as key predictors of less severe COVID-19 outcomes 
Fig. 2 Forest plot of the association between care escalation and race, patients diagnosed with COVID-19 and admitted to Atrium Health Hospital at Home. Legend: Black circle and horizontal line represent the point estimate for each odds ratio and $95 \%$ confidence interval, respectively. Abbreviation: OR, odds ratio; LCI, lower confidence interval; UCL, upper confidence interval; Ref, reference group

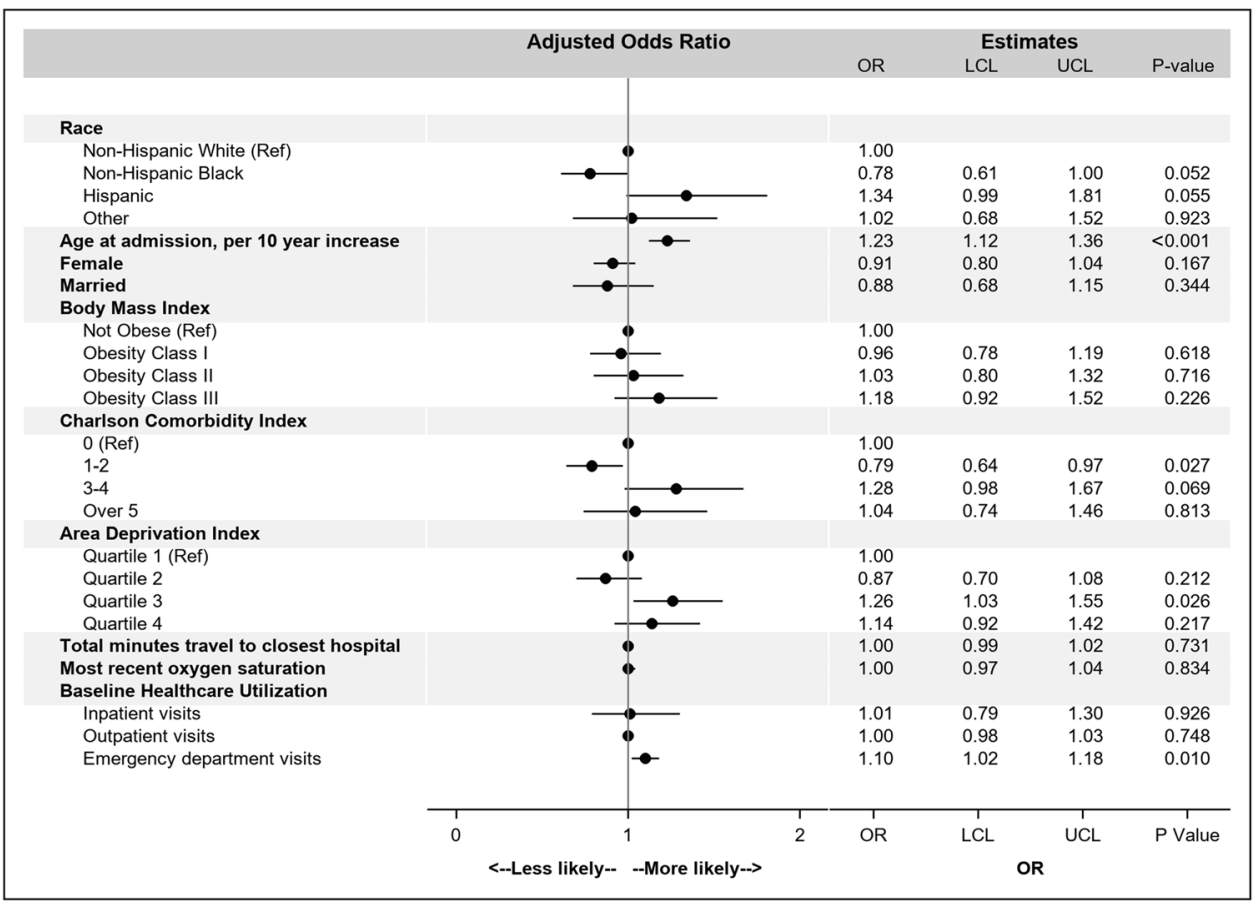

[28]. Additionally, AH-HaH offers access to care that overcomes many traditional barriers that may contribute to published health disparities. While the odds of care escalation were not significant, the direction towards increased risk despite this being a younger, healthier population highlights the potential for social determinants of health, like disadvantaged living and working conditions, to play an outsized role in affecting outcomes. Supporting this notion, we evaluated area level factors (i.e., ADI) as proxies for individual level social determinants and found higher area level deprivation, specifically quartile 3 and to a lesser degree quartile 4, was associated with increased odds of care escalation. We also found patterns suggesting high rates of care escalation with higher area level deprivation within each race/ethnicity strata, though small subgroup sample sizes limit robust interpretation. Additionally, while census tracts aim to represent small, homogenous population groups, there remains substantial potential for individual variation across census tracts and ADI quartiles. Home environment is key to safe and effective delivery of $\mathrm{HaH}$ care. Thus, advancing knowledge of strategies to enhance access to and delivery of $\mathrm{HaH}$ care to patients in diverse living conditions and how $\mathrm{HaH}$ models can partner with support resources to make diverse living conditions safer are key areas for further research.

Furthermore, our study is one of the few studies conducted to investigate racial/ethnicity disparities among patients admitted to a hospital at home program. Prior studies have focused on engagement of minorities in virtual care delivery during the COVID-19 pandemic with mixed results. A study of veterans found that patients who were non-White or of
Hispanic origin tended to be more familiar with virtual care compared to White patients [29]. However, other studies have found that non-Hispanic Blacks tend to use less virtual healthcare compared to Whites, but not Hispanic patients $[30,31]$. Discrepancies between previous findings underscore familiar challenges associated with implementing new care innovations and the importance of diversity and inclusion during early design and evaluation phases. Compared to prior studies with low proportions of Hispanic patients (for example, $1.2 \%$ in study by Aziz et al.) [31], one primary strength of our study was the inclusion of a Hispanic population that was representative of our geographic region (11.1\% vs. $10.7 \%$ in North Carolina) [32]—strengthening the applicability of our findings to the overall population from which the study cohort was drawn. As the healthcare services offered via telemedicine are increasing at an exponential rate, continued evaluation will be needed to determine how different patient groups interact with these services and to consider the potential impact of widespread implementation.

Finally, our study also found that patients with higher numbers of ED visits in the year prior to $\mathrm{AH}-\mathrm{HaH}$ admission were more likely to be transferred to brick-and-mortar hospital within 14 days than others. There are multiple different interpretations that may be relevant to our findings. Patients with higher ED visits prior to $\mathrm{AH}-\mathrm{HaH}$ admission may be more vulnerable to the effects of COVID-19, as these patients tended to have more comorbidities than patients with lower ED visits. Alternatively, higher ED utilization may reflect structural or financial barriers that limit access to needed healthcare services, such as outpatient primary 
Table 2 Characteristics of patients with COVID-19 who received AH-HaH care, stratified by race/ethnicity

\begin{tabular}{|c|c|c|c|c|c|c|c|c|c|}
\hline & \multicolumn{3}{|c|}{ Non-Hispanic White } & \multicolumn{3}{|c|}{ Non-Hispanic Black } & \multicolumn{3}{|l|}{ Hispanic } \\
\hline & \multicolumn{2}{|c|}{ Care escalation within 14 days } & \multirow[t]{2}{*}{$p$-value } & \multicolumn{2}{|c|}{ Care escalation within 14 days } & \multirow[t]{2}{*}{$p$-value } & \multicolumn{2}{|c|}{ Care escalation within 14 days } & \multirow[t]{2}{*}{$p$-value } \\
\hline & No & Yes & & No & Yes & & No & Yes & \\
\hline All & $876(82.7)$ & $183(17.3)$ & & $527(85.0)$ & $93(15.0)$ & & $186(80.9)$ & $44(19.1)$ & \\
\hline $\begin{array}{l}\text { Age at admis- } \\
\text { sion, median } \\
\text { [IQR], years }\end{array}$ & $59[49,69]$ & $63[55,72]$ & $<0.001$ & $51[40,61]$ & $55[46,65]$ & 0.014 & $45.5[37,56]$ & $49[33.5,59]$ & 0.950 \\
\hline Female, $n(\%)$ & $405(46.2)$ & $81(44.3)$ & 0.684 & $306(58.1)$ & $51(54.8)$ & 0.571 & $90(48.4)$ & $21(47.7)$ & 1.000 \\
\hline Married, $n(\%)$ & $620(70.8)$ & $130(71.0)$ & 1.000 & $200(38.0)$ & $32(34.4)$ & 0.562 & $89(47.8)$ & $19(43.2)$ & 0.617 \\
\hline $\begin{array}{l}\text { Body mass } \\
\quad \text { index, } n(\%)\end{array}$ & & & 0.531 & & & 0.417 & & & 0.968 \\
\hline Not obese & $364(41.6)$ & $76(41.5)$ & & $132(25.0)$ & $27(29.0)$ & & $76(40.9)$ & $17(38.6)$ & \\
\hline $\begin{array}{l}\text { Obesity } \\
\text { Class I }\end{array}$ & $258(29.5)$ & $46(25.1)$ & & $140(26.6)$ & $25(26.9)$ & & $68(36.6)$ & $18(40.9)$ & \\
\hline $\begin{array}{l}\text { Obesity Class } \\
\text { II }\end{array}$ & $130(14.8)$ & $33(18.0)$ & & $92(17.5)$ & $10(10.8)$ & & $27(14.5)$ & $6(13.6)$ & \\
\hline $\begin{array}{l}\text { Obesity Class } \\
\text { III }\end{array}$ & $124(14.2)$ & $28(15.3)$ & & 163 (30.9) & $31(33.3)$ & & $15(8.1)$ & $3(6.8)$ & \\
\hline $\begin{array}{l}\text { Charlson Comor- } \\
\text { bidity Index, } \\
n(\%)\end{array}$ & & & $<0.001$ & & & 0.313 & & & 0.235 \\
\hline 0 & $372(42.5)$ & $65(35.5)$ & & $198(37.6)$ & $32(34.4)$ & & $114(61.3)$ & $29(65.9)$ & \\
\hline $1-2$ & $333(38.0)$ & $55(30.1)$ & & $223(42.3)$ & $37(39.8)$ & & $54(29.0)$ & $11(25.0)$ & \\
\hline $3-4$ & $108(12.3)$ & $39(21.3)$ & & $56(10.6)$ & $9(9.7)$ & & $8(4.3)$ & $4(9.1)$ & \\
\hline$\geq 5$ & $63(7.2)$ & $24(13.1)$ & & $50(9.5)$ & $15(16.1)$ & & $10(5.4)$ & $0(0.0)$ & \\
\hline $\begin{array}{l}\text { Area Deprivation } \\
\text { Index, } n(\%)\end{array}$ & & & 0.001 & & & 0.950 & & & 0.847 \\
\hline Quartile 1 & $303(34.6)$ & $43(23.5)$ & & $72(13.7)$ & $13(14.0)$ & & $28(15.1)$ & $8(18.2)$ & \\
\hline Quartile 2 & $228(26.0)$ & $39(21.3)$ & & $151(28.7)$ & $24(25.8)$ & & $47(25.3)$ & $9(20.5)$ & \\
\hline Quartile 3 & $217(24.8)$ & $64(35.0)$ & & $95(18.0)$ & $18(19.4)$ & & $43(23.1)$ & $9(20.5)$ & \\
\hline Quartile 4 & $128(14.6)$ & $37(20.2)$ & & 209 (39.7) & $38(40.9)$ & & $68(36.6)$ & $18(40.9)$ & \\
\hline $\begin{array}{l}\text { Total minutes } \\
\text { travel to the } \\
\text { closest hos- } \\
\text { pital, median } \\
\text { [IQR] }\end{array}$ & $16.7[12.8,23]$ & $17[12.7,22]$ & 0.364 & $13.9[10.4,18.9]$ & $13.7[11,20]$ & 0.747 & $13.8[10.9,19.1]$ & $13.2[9.7,22]$ & 0.451 \\
\hline $\begin{array}{l}\text { Most recent } \mathrm{O}_{2} \\
\text { saturation, } \\
\text { median }[\mathrm{IQR}]\end{array}$ & $95[94,97]$ & $95[94,96.5]$ & 0.193 & $96[94,98]$ & $96[95,98]$ & 0.250 & $96[94,97]$ & $96.5[94.5,98]$ & 0.515 \\
\hline \multicolumn{10}{|l|}{$\begin{array}{l}\text { Baseline health- } \\
\text { care utiliza- } \\
\text { tion*, median } \\
\text { [IQR] }\end{array}$} \\
\hline $\begin{array}{l}\text { Inpatient } \\
\text { visits }\end{array}$ & $0[0,0]$ & $0[0,0]$ & 0.027 & $0[0,0]$ & $0[0,0]$ & 0.388 & $0[0,0]$ & $0[0,0]$ & 0.361 \\
\hline $\begin{array}{l}\text { Outpatient } \\
\text { visits }\end{array}$ & $0[0,2]$ & $0[0,3]$ & 0.091 & $0[0,2]$ & $1[0,3]$ & 0.234 & $0[0,1]$ & $0[0,1.5]$ & 0.736 \\
\hline $\begin{array}{l}\text { Emergency } \\
\text { department } \\
\text { visits }\end{array}$ & $0[0,1]$ & $0[0,1]$ & 0.342 & $1[0,1]$ & $1[0,2]$ & 0.105 & $0[0,1]$ & $1[0,1]$ & 0.102 \\
\hline
\end{tabular}

Abbreviation: $I Q R$, interquartile range

*Baseline healthcare utilization includes all visits within 1 year prior to the index date 
Table 3 Characteristics of patients with COVID-19 who received AH-HaH care, stratified by age group

\begin{tabular}{|c|c|c|c|c|c|c|}
\hline & \multicolumn{3}{|l|}{ Age $<65$} & \multicolumn{3}{|l|}{ Age $\geq 65$} \\
\hline & \multicolumn{2}{|c|}{ Care escalation within 14 days } & \multirow[t]{2}{*}{$p$-value } & \multicolumn{2}{|c|}{ Care escalation within 14 days } & \multirow[t]{2}{*}{$p$-value } \\
\hline & No & Yes & & No & Yes & \\
\hline All & $1238(84.9)$ & $220(15.1)$ & & $448(79.2)$ & $118(20.8)$ & \\
\hline Race/ethnicity, $n(\%)$ & & & 0.343 & & & 0.642 \\
\hline Non-Hispanic White & $563(45.5)$ & $101(45.9)$ & & $313(69.9)$ & $82(69.5)$ & \\
\hline Non-Hispanic Black & $437(35.3)$ & $67(30.5)$ & & $90(20.1)$ & $26(22.0)$ & \\
\hline Hispanic & $164(13.2)$ & $37(16.8)$ & & $22(4.9)$ & $7(5.9)$ & \\
\hline Other or not report & $74(6.0)$ & $15(6.8)$ & & $23(5.1)$ & $3(2.5)$ & \\
\hline Female, $n(\%)$ & $618(49.9)$ & $99(45.0)$ & 0.188 & $229(51.1)$ & $60(50.8)$ & 1.000 \\
\hline Married, $n(\%)$ & $689(55.7)$ & $118(53.6)$ & 0.607 & $282(62.9)$ & $72(61.0)$ & 0.749 \\
\hline Body mass index, $n(\%)$ & & & 0.758 & & & 0.594 \\
\hline Not obese & $371(30.0)$ & $62(28.2)$ & & $253(56.5)$ & $67(56.8)$ & \\
\hline Obesity Class I & 389 (31.4) & $66(30.0)$ & & $101(22.5)$ & $27(22.9)$ & \\
\hline Obesity Class II & $208(16.8)$ & $43(19.5)$ & & $54(12.1)$ & $10(8.5)$ & \\
\hline Obesity Class III & $270(21.8)$ & $49(22.3)$ & & $40(8.9)$ & $14(11.9)$ & \\
\hline Charlson Comorbidity Index, $n(\%)$ & & & 0.092 & & & 0.022 \\
\hline 0 & $606(48.9)$ & $100(45.5)$ & & $126(28.1)$ & $34(28.8)$ & \\
\hline $1-2$ & $480(38.8)$ & $81(36.8)$ & & $169(37.7)$ & $29(24.6)$ & \\
\hline $3-4$ & $93(7.5)$ & $28(12.7)$ & & $83(18.5)$ & $26(22.0)$ & \\
\hline$\geq 5$ & $59(4.8)$ & $11(5.0)$ & & $70(15.6)$ & $29(24.6)$ & \\
\hline Area Deprivation Index, $n(\%)$ & & & 0.230 & & & 0.062 \\
\hline Quartile 1 & $298(24.1)$ & $48(21.8)$ & & $134(29.9)$ & $23(19.5)$ & \\
\hline Quartile 2 & $344(27.8)$ & $50(22.7)$ & & $113(25.2)$ & $27(22.9)$ & \\
\hline Quartile 3 & $270(21.8)$ & $57(25.9)$ & & $102(22.8)$ & $36(30.5)$ & \\
\hline Quartile 4 & $326(26.3)$ & $65(29.5)$ & & $99(22.1)$ & $32(27.1)$ & \\
\hline $\begin{array}{l}\text { Total minutes travel to the closest hospital, median } \\
\text { [IQR] }\end{array}$ & $15.5[11.5,21.6]$ & $15.5[11.6,21.8]$ & 0.697 & $16.2[12.2,21.8]$ & $15.5[11.6,21.4]$ & 0.322 \\
\hline Most recent $\mathrm{O}_{2}$ saturation, median [IQR] & $96[94,98]$ & $96[94,97]$ & 0.720 & $95[94,97]$ & $96[94,97]$ & 0.792 \\
\hline \multicolumn{7}{|l|}{ Baseline healthcare utilization*, median [IQR] } \\
\hline Inpatient visits & $0[0,0]$ & $0[0,0]$ & 0.302 & $0[0,0]$ & $0[0,0]$ & 0.128 \\
\hline Outpatient visits & $0[0,1]$ & $0[0,2]$ & 0.143 & $1[0,3]$ & $1[0,4]$ & 0.365 \\
\hline Emergency department visits & $0[0,1]$ & $1[0,1]$ & 0.168 & $0[0,1]$ & $0.5[0,1]$ & 0.041 \\
\hline
\end{tabular}

Abbreviation: $I Q R$, interquartile range

*Baseline healthcare utilization includes all visits within 1 year prior to the index date

care, and increase risk of poor health outcomes like disease progression requiring care escalation. Similar underpinning reasons as are likely to be present for patients residing in areas with a high neighborhood deprivation index.

\section{Limitation}

Several limitations should be noted in this study. First, this retrospective study was conducted within a defined region of southeastern U.S., which may limit generalizability to regions with different populations, healthcare infrastructure, or COVID-19 policies. However, we include a large, diverse cohort from urban and rural areas. Of particular note, regarding the generalizability of our findings, there have been large regional differences observed in COVID-19 disparities [33, 34]. Indeed, our findings that non-Hispanic Blacks did not experience worse care escalation outcomes and may even have had better outcomes, compared to non-Hispanic Whites, may provide further evidence in support of these regional differences. Second, there may be unique aspects of the AH-HaH delivery model compared to other Hospital at Home programs, and generalizability may be further limited to patients meeting the $\mathrm{AH}-\mathrm{HaH}$ eligibility criteria (e.g., a working telephone, either patient or family members who speak English or Spanish, and ability to comply with monitoring protocols). Third, while we included area-level demographics (i.e., ADI and total minutes travel to the closest hospital) as a proxy estimation of socioeconomic status, 
we lacked the additional precision that would be gained from direct measures of patients' socioeconomic status that may affect patient outcomes.

\section{Conclusion}

Our results suggest that $\mathrm{AH}-\mathrm{HaH}$ effectively leveraged telemedicine to reach a diverse population, reinforcing that such models can play a key role in advancing access to care. However, the higher odds of care escalation for patients living in areas with high area deprivation indexes highlight the need both for novel programs to vigilantly monitor disparities and for further research that explores if tailored support like enhanced social services may ensure that vulnerable populations are able to access novel care strategies.

\section{Role of the Funder/Sponsor}

The funders had no role in the design and conduct of the study; collection, management, analysis, and interpretation of the data; preparation, review, or approval of the manuscript; and decision to submit the manuscript for publication.

Acknowledgements The authors thank the numerous teammates at Atrium Health whose tireless efforts, innovative thinking, and leadership helped make $\mathrm{AH}-\mathrm{HaH}$ a viable care option for our patients during the COVID-19 pandemic. Special thanks to the data management and project management teams for their efforts in coordinating and developing the database used for this research.

Author Contribution Drs. Liu and Chou had full access to all of the data in the study and take responsibility for the integrity of the data and the accuracy of the data analysis. Concept and design: Liu, Chou, Kowalkowski. Acquisition, analysis, or interpretation of data: Liu, Chou, Taylor, Kowalkowski, Priem, Sitammagari, McWilliams. Drafting of the manuscript: Liu, Chou, Taylor. Critical revision of the manuscript for important intellectual content: Liu, Chou, Taylor, Murphy, Kowalkowski, Priem, Sitammagari, McWilliams. Statistical analysis: Liu, Chou. Administrative, technical, or material support: Murphy, Hole, McWilliams. Supervision: Taylor, McWilliams.

Funding The study was funded by Atrium Health.

\section{Declarations}

Ethics Approval This study was approved by the Atrium Health Institutional Review Board.

Informed Consent Not applicable.

Consent to Participate Not applicable; this was a retrospective cohort study.

Consent for Publication Not applicable; this was a retrospective cohort study.
Conflict of Interest The authors declare no competing interests.

\section{References}

1. WHO Coronavirus (COVID-19) Dashboard [Internet]. World Health Organization. 2021. https://covid19.who.int/. Accessed Feb 162022

2. COVID Data Tracker [Internet]. Centers for Disease Control and Prevention. 2021. https://covid.cdc.gov/covid-data-tracker/\#datat racker-home. Accessed Feb 162022

3. Yu Q, Wu X, Li B, Scribner RA. Multiple mediation analysis with survival outcomes: with an application to explore racial disparity in breast cancer survival. Stat Med. 2019;38(3):398-412. https:// doi.org/10.1002/sim.7977.

4. Yeter D, Banks EC, Aschner M. Disparity in risk factor severity for early childhood blood lead among predominantly AfricanAmerican Black children: the 1999 to 2010 US NHANES. Int J Environ Res Public Health. 2020;17(5):1552. https://doi.org/10. 3390/ijerph17051552.

5. Gupta V, Haque I, Chakraborty J, Graff S, Banerjee S, Banerjee SK. Racial disparity in breast cancer: can it be mattered for prognosis and therapy. J Cell Commun Signal. 2018;12(1):119-32. https://doi.org/10.1007/s12079-017-0416-4.

6. Chang SH, Yu YC, Carlsson NP, Liu X, Colditz GA. Racial disparity in life expectancies and life years lost associated with multiple obesity-related chronic conditions. Obesity (Silver Spring). 2017;25(5):950-7. https://doi.org/10.1002/oby.21822.

7. Bowblis JR, Ng W, Akosionu O, Shippee TP. Decomposing racial and ethnic disparities in nursing home quality of life. J Appl Gerontol. 2021;40(9):1051-61. https://doi.org/10.1177/0733464820946659.

8. McCree DH, Chesson H, Bradley ELP, Williams A, Gant Z, Geter A. Exploring changes in racial/ethnic disparities of HIV diagnosis rates under the "Ending the HIV Epidemic: A Plan for America" Initiative. Public Health Rep. 2020;135(5):685-90. https://doi.org/ 10.1177/0033354920943526.

9. Dai CL, Kornilov SA, Roper RT, Cohen-Cline H, Jade K, Smith $\mathrm{B}$, et al. Characteristics and factors associated with COVID-19 infection, hospitalization, and mortality across race and ethnicity. Clin Infect Dis. 2021. https://doi.org/10.1093/cid/ciab154.

10. Renelus BD, Khoury NC, Chandrasekaran K, Bekele E, Briggs WM, Ivanov A, et al. Racial disparities in COVID-19 hospitalization and in-hospital mortality at the height of the New York City pandemic. J Racial Ethn Health Disparities. 2021;8(5):1161-7. https://doi.org/10.1007/s40615-020-00872-x.

11. Gu T, Mack JA, Salvatore M, Prabhu Sankar S, Valley TS, Singh $\mathrm{K}$, et al. Characteristics associated with racial/ethnic disparities in COVID-19 outcomes in an academic health care system. JAMA Netw Open. 2020;3(10):e2025197. https://doi.org/10.1001/jaman etworkopen.2020.25197.

12. Asfaw A. Racial disparity in potential occupational exposure to COVID-19. J Racial Ethn Health Disparities. 2021. https://doi. org/10.1007/s40615-021-01110-8.

13. Anyane-Yeboa A, Sato T, Sakuraba A. Racial disparities in COVID-19 deaths reveal harsh truths about structural inequality in America. J Intern Med. 2020;288(4):479-80. https://doi.org/ 10.1111/joim.13117.

14. Nouri S, Khoong EC, Lyles CR, Karliner L. Addressing equity in telemedicine for chronic disease management during the COVID19 pandemic. NEJM Catal Innov Care Deliv 2020;1(3). https:// doi.org/10.1056/CAT.20.0123

15. Balatbat C, Kadakia KT, Dzau VJ, Offodile AC. No place like home: hospital at home as a post-pandemic frontier for care delivery innovation. NEJM Catal Innov Care Deliv. 2021;4:1-6. https:// doi.org/10.1056/CAT.21.0237. 
16. Johnson EE. Acute care reimagined: home hospital care can support the triple aim and reduce health disparities. J Healthc Manag. 2021;66(4):258-70. https://doi.org/10.1097/JHM-D-21-00174.

17. CMS. Acute hospital care at home resources. 2021. https://quali tynet.cms.gov/acute-hospital-care-at-home/resources. Accessed 3 Jan 2022

18. Sitammagari K, Murphy S, Kowalkowski M, Chou S-H, Sullivan $\mathrm{M}$, Taylor S, et al. Insights from rapid deployment of a "Virtual Hospital" as standard care during the COVID-19 pandemic. Ann Intern Med. 2021;174(2):192-9. https://doi.org/10.7326/ $\mathrm{m} 20-4076$.

19. Chou S-H, McWilliams A, Murphy S, Sitammagari K, Liu T-L, Hole $\mathrm{C}$, et al. Factors associated with risk for care escalation among patients with COVID-19 receiving home-based hospital care. Ann Intern Med. 2021. https://doi.org/10.7326/m21-0409.

20. Kind AJH, Buckingham WR. Making neighborhood-disadvantage metrics accessible - the neighborhood atlas. N Engl J Med. 2018;378(26):2456-8.

21. Singh GK. Area deprivation and widening inequalities in US mortality, 1969-1998. Am J Public Health. 2003;93(7):1137-43.

22. 2013-2017 ACS 5-year estimates [Internet]. United States Census Bureau. 2021. https://www.census.gov/programs-surveys/acs/ technical-documentation/table-and-geography-changes/2017/5year.html. Accessed Jan 72022

23. Nau C, Bruxvoort K, Navarro RA, Chevez SG, Hogan TA, Ironside KR, et al. COVID-19 inequities across multiple racial and ethnic groups: results from an integrated health care organization. Ann Intern Med. 2021. https://doi.org/10.7326/M20-8283.

24. Velasco F, Yang DM, Zhang M, Nelson T, Sheffield T, Keller T, et al. Association of healthcare access with intensive care unit utilization and mortality in patients of hispanic ethnicity hospitalized with COVID-19. J Hosp Med. 2021;16(11):659-66. https:// doi.org/10.12788/jhm.3717.

25. Cervantes J, Sureen A, Galura G, Dodoo C, Dwivedi AK, Bashashati M, et al. Factors associated with COVID-19 severity and mortality among Hispanic patients living on the USA-Mexico border. J Investig Med. 2021;69:819-23.

26. Macias Gil R, Touzard-Romo F, Sanchez MC, Pandita A, Kalligeros M, Mylona EK, et al. Characteristics and outcomes of Hispanic/Latinx patients with coronavirus disease 19 (COVID-19) requiring hospitalization in Rhode Island: a retrospective cohort study. Ann Epidemiol. 2021;58:64-8. https://doi.org/10.1016/j. annepidem.2021.03.003.
27. Macias Gil R, Marcelin JR, Zuniga-Blanco B, Marquez C, Mathew T, Piggott DA. COVID-19 pandemic: disparate health impact on the Hispanic/Latinx population in the United States. J Infect Dis. 2020;222(10):1592-5. https://doi.org/10.1093/infdis/ jiaa474.

28. Wynants L, Van Calster B, Collins GS, Riley RD, Heinze G, Schuit E, et al. Prediction models for diagnosis and prognosis of covid-19: systematic review and critical appraisal. BMJ. 2020;369:m1328. https://doi.org/10.1136/bmj.m1328.

29. Ferguson JM, Jacobs J, Yefimova M, Greene L, Heyworth L, Zulman DM. Virtual care expansion in the Veterans Health Administration during the COVID-19 pandemic: clinical services and patient characteristics associated with utilization. J Am Med Inform Assoc. 2021;28(3):453-62. https://doi.org/10.1093/jamia/ ocaa284.

30. Abdel-Rahman O. Patient-related barriers to some virtual healthcare services among cancer patients in the USA: a populationbased study. J Comp Eff Res. 2021;10(2):119-26.

31. Aziz K, Moon JY, Parikh R, Lorch AC, Friedman DS, Miller $\mathrm{JB}$, et al. Association of patient characteristics with delivery of ophthalmic telemedicine during the COVID-19 pandemic. JAMA Ophthalmol. 2021;139(11):1174-82. https://doi.org/10.1001/ jamaophthalmol.2021.3728.

32. North Carolina's Hispanic Community: 2021 Snapshot [Internet]. Carolina Demography: UNC Carolina Population Center. 2021. https://www.ncdemography.org/2021/10/18/north-carolinas-hispa nic-community-2021-snapshot/?utm_source=Carolina+Popul ation+Center\&utm_campaign $=2 \mathrm{~d} 98 \mathrm{a} 02 \mathrm{e} 60$-EMAIL_CAMPA IGN_2021_10_25_06_38\&utm_medium $=$ email\&utm_term $=0$ _ 807b260e90-2d98a02e60-36743817. Accessed Dec 212021

33. Ferguson JM, Justice AC, Osborne TF, et al. Geographic and temporal variation in racial and ethnic disparities in SARSCoV-2 positivity between February 2020 and August 2021 in the United States. Sci Rep. 2022;12(1):273. https://doi.org/10.1038/ s41598-021-03967-5.

34. Romano SD, Blackstock AJ, Taylor EV, et al. Trends in racial and ethnic disparities in COVID-19 hospitalizations, by region - United States, March-December 2020. Morbid Mortal Wkly Rep. 2021;70(15):560-5. https://doi.org/10.15585/mmwr.mm701 $5 \mathrm{e} 2$.

Publisher's Note Springer Nature remains neutral with regard to jurisdictional claims in published maps and institutional affiliations. 